\title{
Archéopages
}

Archéopages

Archéologie et société

Hors-série 2 | 2010

Archéologie sans frontières

\section{Cuivre, laiton, dinanderie mosane : ateliers et productions métallurgiques à Dinant et Bouvignes du XIII ${ }^{\mathrm{e}} \mathrm{au} \mathrm{XVI}{ }^{\mathrm{e}}$ siècle}

Nicolas Thomas et Jean Plumier

\section{C) OpenEdition}

1 Journals

Édition électronique

URL : https://journals.openedition.org/archeopages/823

DOI : 10.4000/archeopages.823

ISSN : 2269-9872

Éditeur

INRAP - Institut national de recherches archéologiques préventives

Édition imprimée

Date de publication : 1 octobre 2010

Pagination : 142-151

ISSN : 1622-8545

Référence électronique

Nicolas Thomas et Jean Plumier, « Cuivre, laiton, dinanderie mosane : ateliers et productions

métallurgiques à Dinant et Bouvignes du xIII au xvi siècle ", Archéopages [En ligne], Hors-série 2 |

2010, mis en ligne le 01 octobre 2010, consulté le 23 février 2023. URL : http://

journals.openedition.org/archeopages/823; DOI : https://doi.org/10.4000/archeopages.823

Tous droits réservés 


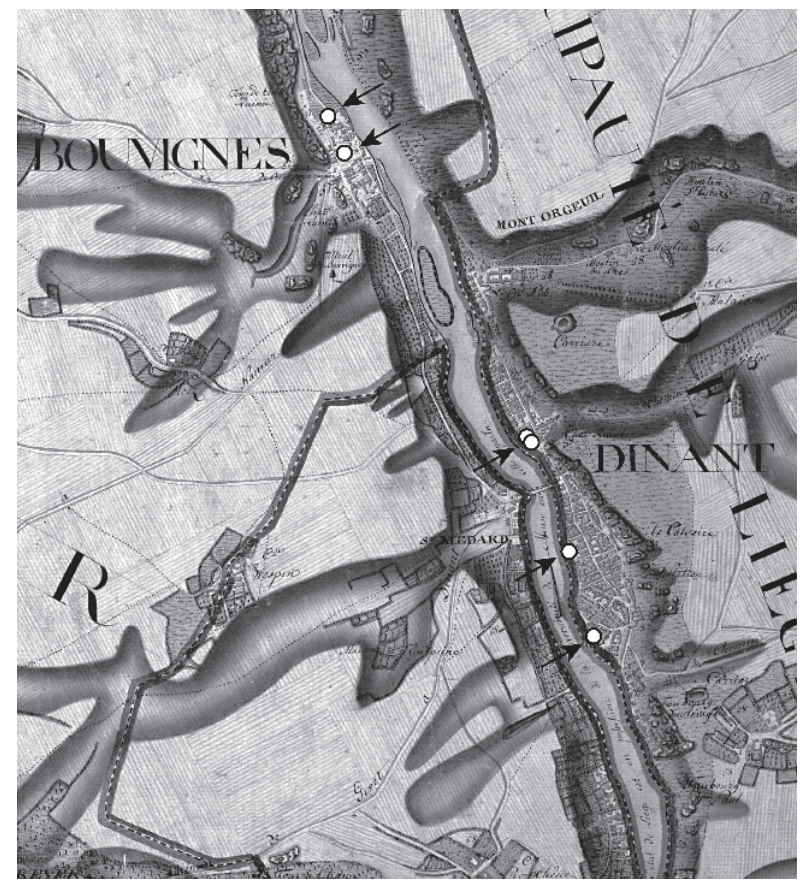

\section{出}

$¥$

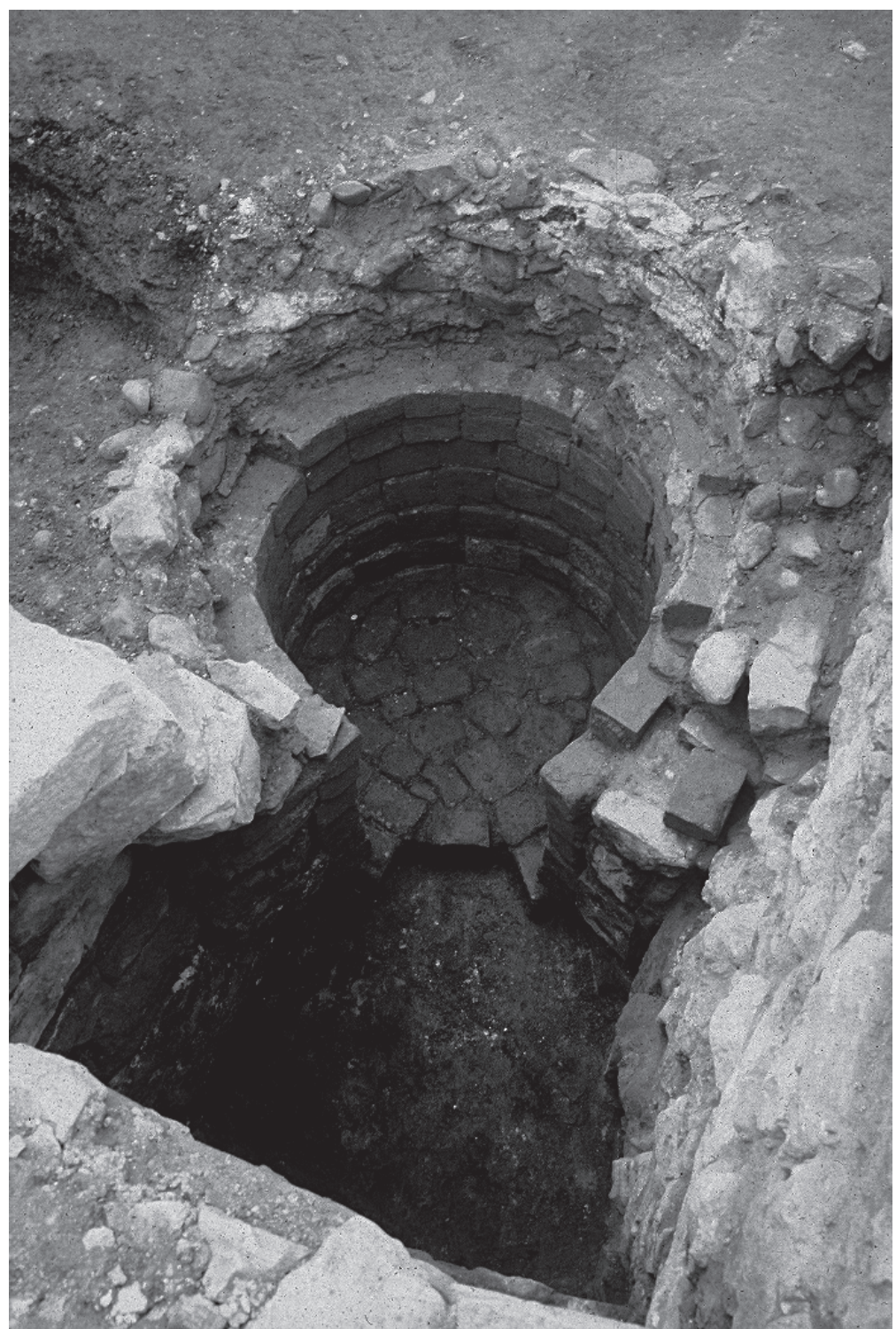

[Fig. 1] Dinant (au sud) et Bouvignes (au nord) sur l'atlas dit de Ferraris (1774). Localisation des fouilles

archéologiques. Bouvignes dépend du comté de Namur tandis que

Dinant est sous l'autorité du princeévêque de Liège. À la fin du

XIII ${ }^{\mathrm{e}}$ siècle, les deux villes, séparées par la Meuse, sont situées à la

frontière entre le comté et la

principauté, situation à l'origine

de la concurrence industrielle et

commerciale entre les deux voisines.
[Fig.2] Un des fours de fusion découvert à Bouvignes, porte Chevalier

$\left(\mathrm{xV}^{\mathrm{e}}\right.$ siècle). Les briques réfractaires de la partie supérieure du four ont été récupérées, probablement

pour construire un autre four. Les fondeurs ont utilisé le rempart pour construire ce four très profond. 


\section{Cuivre, laiton, dinanderie mosane : ateliers et productions métallurgiques à Dinant et Bouvignes du XIII ${ }^{\mathrm{e}}$ au XVI ${ }^{\mathrm{e}}$ siècle}

\section{Nicolas Thomas}

Inrap - Centre-île-de-France, Laboratoire de médiévistique occidentale de Paris (LAMOP), UMR 8589, CNRS-Université Paris 1 Panthéon-Sorbonne, Équipe d'histoire des techniques Jean Plumie

Service public de Wallonie, directeur de l'archéologie, département du Patrimoine, $\mathrm{DGO}_{4}$

$\mathrm{L}$ e contexte scientifique. Alors que le cuivre et ses alliages se trouvent partout au Moyen Âge, dans la cuisine, sur la table, dans les églises et même sur le costume, le travail du cuivre à cette période demeure particulièrement méconnu aujourd'hui (Thomas, 2010). Comment les alliages sont-ils élaborés? Quelles sont les techniques de mise en forme du métal ? Quelle est l'organisation des ateliers et comment ces moyens de production ont-ils permis une diffusion si importante de ces produits à la fois dans l'ensemble de l'Europe médiévale, mais aussi dans toutes les couches de la société? Les croissances démographique et économique qui caractérisent les premiers siècles après l'an Mil et la concentration des hommes dans les villes s'accompagnent d'une forte demande en produits manufacturés, ce qui entraîne l'intensification de l'exploitation des mines, surtout dans l'est de l'Europe. À la fin du XIII ${ }^{\mathrm{e}}$ siècle, fait exclusivement urbain, les artisans s'organisent en métier et l'on observe une division du travail accrue, certaines villes se spécialisent même dans de quasi mono-industries. Il en est ainsi de deux villes voisines, Dinant et Bouvignes, le long de la Meuse belge qui, toutes deux, vont se tourner vers la production de récipients en cuivre, poêles et chaudrons, et le commerce à longue distance. Les marchands dinantais et bouvignois inondent l'Occident médiéval de leurs productions, dont le royaume de France, où ils sont très actifs, en particulier sur les marchés et foires de Champagne, de Bourgogne, d'Anjou, de Touraine, de Normandie et de Saint-Denis. Au Moyen Âge déjà, la réputation de Dinant est telle que la ville devient éponyme de la vaisselle de cuivre, la « dinanderie», et que le chaudronnier se dénomme « dinandier».
Entre 1995 et 2009, le Service public de Wallonie (SPW) a réalisé deux fouilles à Bouvignes et trois à Dinant sur une vingtaine d'ateliers datés entre le $\mathrm{XIII}^{\mathrm{e}}$ et le $\mathrm{XVI}^{\mathrm{e}}$ siècle, toutes dans le contexte de l'archéologie préventive [Fig.1]. Certains de ces sites montrent une stratigraphie ininterrompue d'activité métallurgique intense sur plusieurs siècles (Plumier, Berckmans, 1996 ; Verbeek, 2007). L'abondance du mobilier récolté et les difficultés liées à l'interprétation de déchets métallurgiques méconnus et non encore étudiés posent des problèmes méthodologiques inédits. L'étude, par l'Inrap, d'un atelier parisien du XIV ${ }^{\mathrm{e}}$ siècle comparable aux ateliers mosans a conduit à un rapprochement des principaux acteurs de ces fouilles archéologiques (Thomas, 2009; Thomas, Bourgarit, 2006). Dès 2007, des contacts informels ont permis notamment l'implication d'un agent de l'Inrap dans la muséographie dédiée à la dinanderie dans la Maison du patrimoine médiéval mosan à Bouvignes, inaugurée en 2008 . L'intérêt pour la richesse exceptionnelle des sites mosans dépassant largement les frontières belges, une convention de partenariat a été signée entre l'Inrap et le Spw à la fin de l'année 2009, afin de mener un programme de recherche sur trois ans, entre 2010 et 2012, devant aboutir à la publication des sites et à un projet muséographique². Les principaux objectifs scientifiques de ce programme visent à définir la nature et l'ampleur des productions, à restituer les chaînes opératoires et à approcher l'organisation des ateliers en les replaçant dans leurs contextes économique et social. Outre l'étude des résultats de fouille, l'ambition des porteurs du programme est de s'intéresser également à la diffusion des produits mosans au travers de sites archéologiques en France ou dans les collections des musées belges ou français. Enfin, ce programme pluridisciplinaire, incluant recherches archéologiques et historiques, sciences des matériaux et études paléo-environnementales, s'appuie sur les acquis méthodologiques de la fouille parisienne avec l'ambition de les développer. L'enjeu dépasse celui de l'expertise apportée par l'Inrap puisque, au-delà des questions scientifiques posées, le programme vise aussi à améliorer les approches utilisées dans l'étude d'une métallurgie jusque-là délaissée, par exemple par des méthodes d'enregistrement spécifiques du mobilier archéologique ou en automatisant des procédés d'analyses des matériaux, que ce soit des métaux, des scories ou des céramiques réfractaires comme les moules de fonderie ou les creusets.

(SPW), Fabienne Pigière (IRScNB), Stéphane Pirson (Spw), Simo Spassov (CgG-Irm), Jacques Toussaint

(MAAN), Françoise Urban (restauratrice métal), Claire-Marie Vandermensbrugghe (MPMM), Laurent Verslype (Université catholique de Louvain) et Jean-Marie Welter (métallurgiste).
Les premiers résultats : une historiographie mise à mal. En 2010, première année du programme, les travaux se sont concentrés sur l'inventaire du mobilier des premiers sites fouillés, sur des analyses de composition d'artéfacts en alliages à base de cuivre et sur la reconstitution expérimentale de techniques de fonderie, d'élaboration de laiton ou encore de fours de fusion. Les premiers résultats mettent à mal l'historiographie qui fait de la présence de minerais de zinc dans la vallée de la Meuse la principale raison déterminant le succès de l'industrie

du cuivre à Dinant et à Bouvignes (Yante, 1988;

(1)

college of London), Goemaere (Institut roya Saint-Luc de Liège, Joseph Hus (Centre Bodart (Archives de globe-CgGiRM), Albert l'État à Namur), David Lemeunier (Université Bourgarit (Centre de Liège), Daniel Pacyna Viviane Bechoux des sciences naturelles

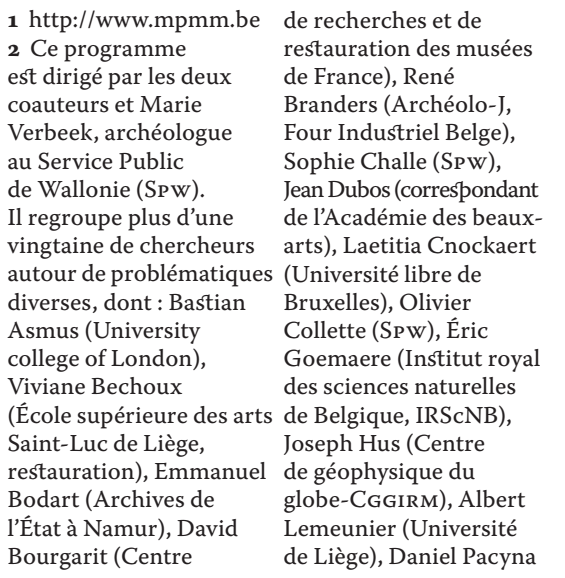




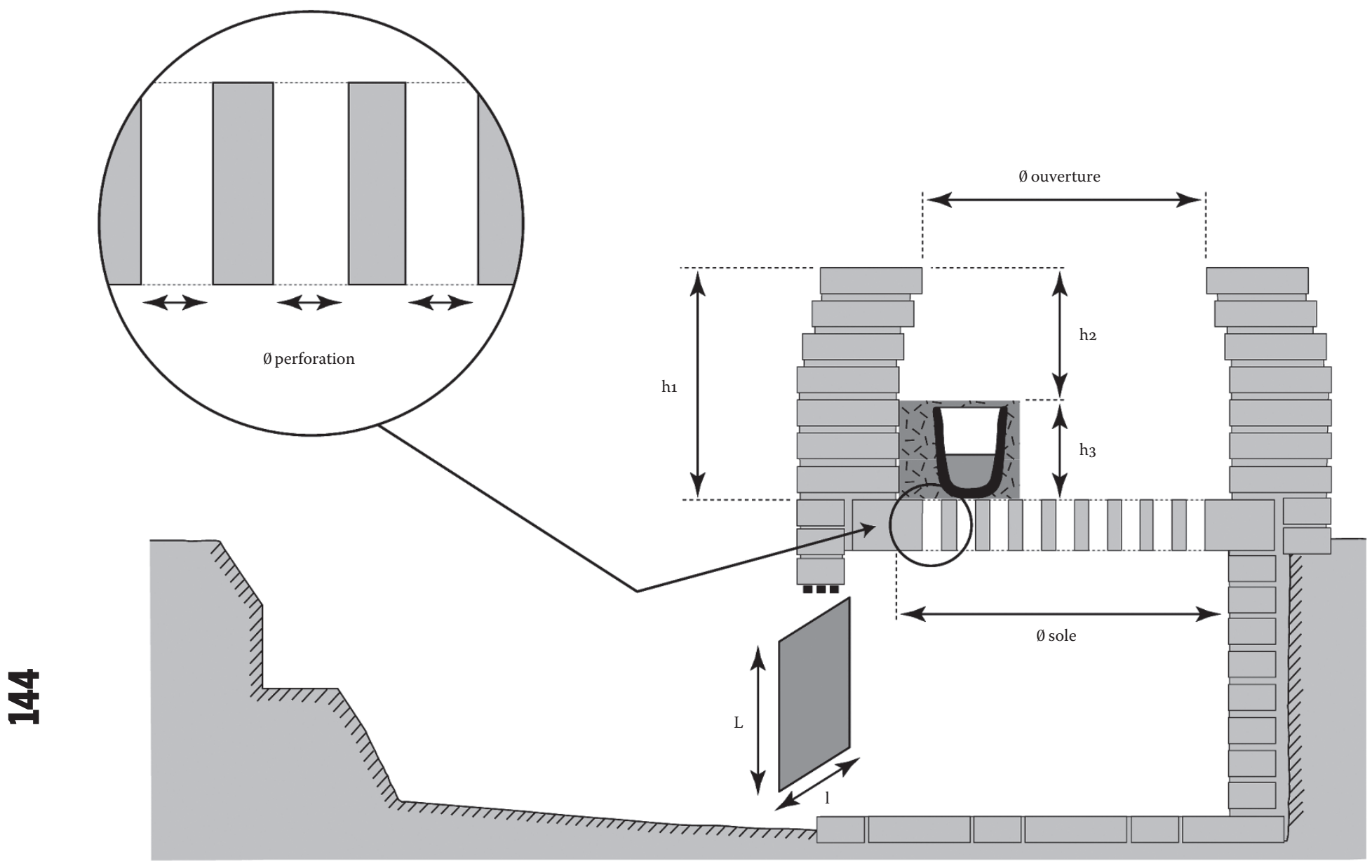

[Fig.3] Schéma d'un four de fusion à ventilation naturelle. Modèle théorique avec les principaux paramètres architecturaux responsables du bon fonctionnement et des températures atteintes dans la chambre de combustion où sont placés les creusets. D'après les textes, les sources archéologiques et les reconstitutions expérimentales, la hauteur de la chambre de combustion dépend de la hauteur des creusets selon un rapport qui n'est pas constant $\left(\mathrm{h}_{2}=\mathrm{xh}_{3}\right.$ : $\left.h_{1}=h_{2}+h_{3} ; h_{1}=x_{3}+h_{3}\right)$. 
Pirenne, 1951a et 1951b). Le laiton est un alliage de cuivre et de zinc, de couleur jaune comme l'or, élaboré directement à partir d'un carbonate de zinc présent en abondance depuis le Limbourg jusque dans la province de Namur. Certes, l'histoire conserve la mémoire de grands fondeurs dinantais et d'œuvres prestigieuses en laiton qui ornent encore les églises ou les musées (Toussaint, 2005 ; Collon-Gevaert, 1951). Bien sûr, les dernières analyses réalisées montrent bien la présence du laiton à la fois dans les ateliers, mais aussi dans la production, notamment dans des chandeliers ou de la vaisselle de table, par opposition à celle qui reste confinée à la cuisine. Toutefois, la dinanderie réservée à la cuisine n'est pas en laiton, du moins celle qui est fondue. La quasi-totalité des chaudrons par exemple, sont des alliages de cuivre qui ne contiennent absolument pas de zinc. Ce sont des bronzes au plomb, c'est-à-dire des cuivres alliés à un peu d'étain et surtout beaucoup de plomb, de 6 à $30 \%$ en masse. Si, pour le moment, le corpus des analyses de composition demeure limité à une cinquantaine d'artéfacts, il est remarquable de constater que ces premières conclusions sont en accord avec ce que l'on a déterminé à partir des ensembles français issus de l'archéologie préventive : les fondeurs et batteurs mosans semblent utiliser les mêmes alliages et choisir leurs compositions non seulement en fonction de contraintes techniques, mais aussi selon des critères économiques (Thomas, 2009, p. 598-625; Bourgarit, Thomas, 2009). Ce sont les mêmes gammes d'alliages auxquelles les artisans mosans ont recours pour réaliser des productions à bas coût susceptibles d'être exportées partout en Europe (Dungworth, Nicholas, 2004; Drescher, 1982). Linventaire des déchets de fonderie provenant des sites fouillés, qui n'en est qu'à ces débuts, rappelons-le, confirmerait ces premiers résultats : l'archéologie rend compte de la production la plus ordinaire au détriment des produits aristocratiques pour lesquels le laiton est certainement l'alliage le plus employé. Les 10 ooo fragments de moules de fonderie en terre inventoriés à ce jour, représentant plusieurs centaines de kilogrammes, témoignent quasiment exclusivement d'une production de chaudrons coulés, produits aux XIV $\mathrm{XI}^{\mathrm{e}}$ et $\mathrm{XV}^{\mathrm{e}}$ siècles, et donc d'une activité où le zinc est absent. Seuls quelques contextes archéologiques du Xve siècle fouillés à Dinant ont livré des fragments de moules de petits chandeliers, mais les chaudrons demeurent la production la plus représentée dans les deux villes. Les premières comparaisons entre les ateliers de Bouvignes et ceux de Dinant montrent par ailleurs qu'il n'y a pas de différence dans les techniques de moulage et de fonderie, tout en suggérant une standardisation de ces techniques permettant une production de masse. À la lueur de ces premiers résultats, il apparaît que la place du laiton dans cette métallurgie doit être réévaluée pour expliquer le succès de Dinant et de Bouvignes. La proximité des mines de calamine a sans aucun doute contribué à l'industrialisation et à la spécialisation des deux cités, mais d'autres facteurs de cette réussite mosane - qu'ils soient liés à d'autres matières premières comme la « derle ", une terre hautement réfractaire, l'abondance de combustible, la qualité des voies de circulation avec le fleuve, les conditions politiques, l'organisation sociale des métiers ou encore le dynamisme des marchands mosans - sont vraisemblablement à mettre plus en avant.

Les fours de fonderie, de l'étude archéologique à l'expérimentation. Les travaux concernant l'interprétation des fours de fusion par l'étude des structures archéologiques et leur reconstitution ont fourni le plus de résultats concrets car s'appuyant sur des travaux antérieurs (Thomas, 2009, p. 759-804). Ce sont plusieurs dizaines de fours qui ont été découverts lors des fouilles à Dinant et à Bouvignes [Fig.2]. Dans ces deux villes se concentrent bien plus de structures de ce type que celles fouillées dans toute l'Europe depuis 30 ans. Ce seul constat montre l'intérêt d'étudier ces fours qui ont été, pour la plupart, incompris. Ils présentent souvent les mêmes caractéristiques : il ne subsiste, lors de la fouille, que la partie en fondation et le creusement en forme de trou de serrure. La fosse est composée de deux parties : l'une, cylindrique, plus rarement carrée, est construite en briques réfractaires et correspond à l'aire de chauffe proprement dite. Il manque évidemment au mieux la partie en élévation, mais le plus souvent les structures sont arasées, ce qui rend malaisé la restitution de leur hauteur jusqu'au niveau de sol de l'atelier et au-delà.

Un modèle théorique éprouvé. À partir de la fouille parisienne, en mêlant les sources archéologiques et les sources écrites, un modèle de fonctionnement général a pu être proposé allant à l'encontre des hypothèses généralement admises jusque-là. Ces fours de fusion fonctionnent sans soufflet, utilisant le principe de la convection, le foyer étant disposé sur une sole perforée placée au milieu du cylindre et les creusets contenant le métal sont placés directement au milieu du charbon de bois. Lors de la mise en chauffe, la masse volumique de l'air au-dessus de la combustion diminue par rapport à l'air ambiant. L'air au-dessus du foyer s'élevant, une dépression est alors créée sous la sole perforée, ce qui entraîne de l'air froid. Plus l'air est chaud, plus il monte vite et plus l'air pénètre par la partie basse sous la sole assurant ainsi naturellement la ventilation du foyer et la montée en température : c'est le tirage. Dans un tel système, la fonction de la sole perforée est double : elle alimente la chambre de combustion avec de l'air, mais elle permet aussi à la cendre et aux plus petits charbons de tomber dans la partie basse, qui est appelée le cendrier. Ainsi la ventilation est améliorée par l'évacuation des cendres et la combustion des plus gros charbons s'en trouve 


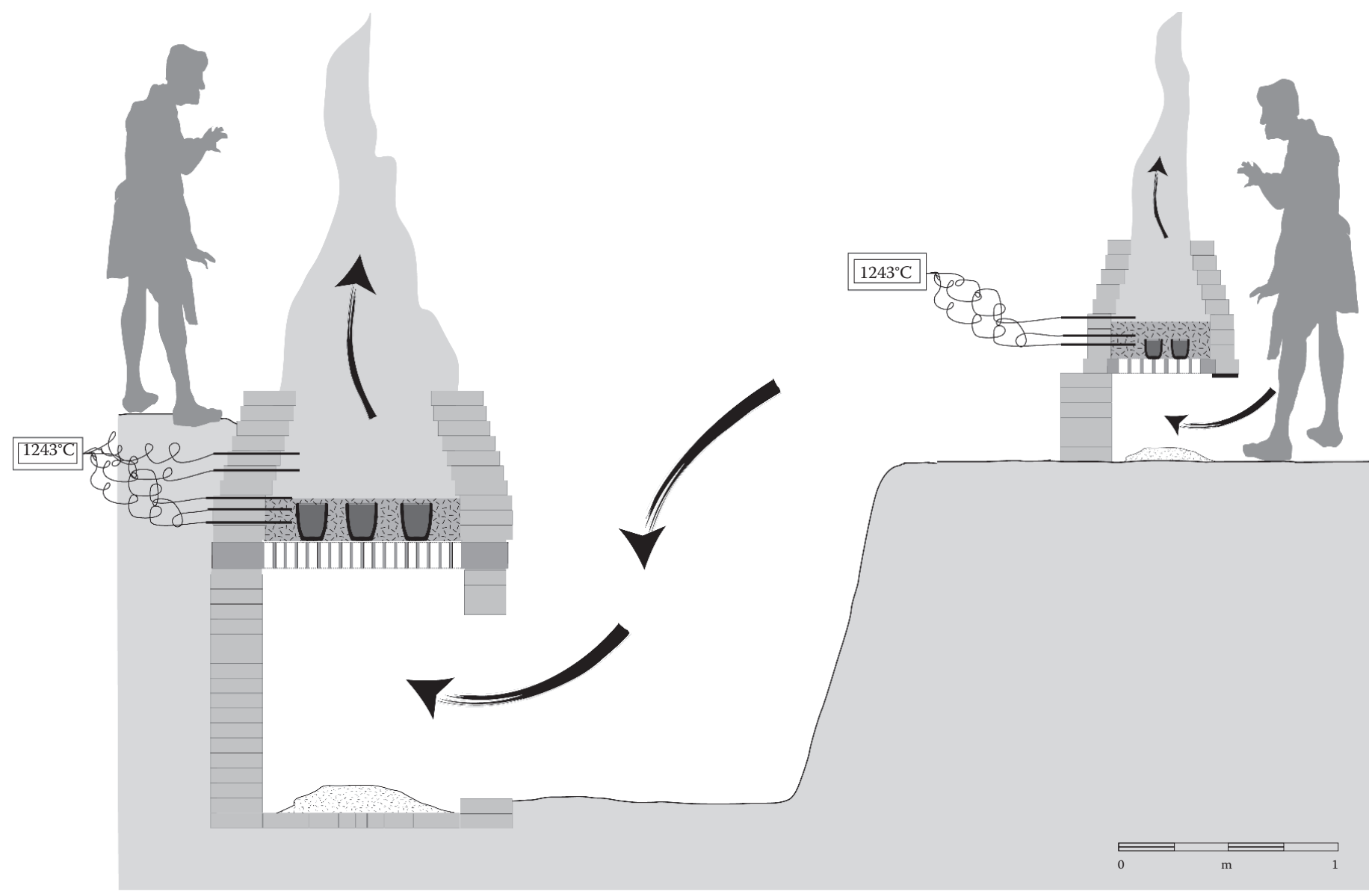

Grand four

Petit four

[Fig.4] Schéma en coupe des deux fours reconstitués et détail de la chambre de combustion avec l'emplacement des thermocouples (Type S : platinerhodium/platine). Chaque

thermocouple est relié via une interface $a d$ hoc à un ordinateur portable permettant un enregistrement des mesures, ici toutes les 5 secondes. Dans le bas de la figure, histogrammes des mesures en degrés Celsius. Pour le grand four, on observe un gradient

thermique très important dans

l'épaisseur du combustible (en gris

sur le schéma). Dans le petit four,

la distribution des mesures montre

une meilleure homogénéité.

Au-dessus, la vitesse de l'air est

tellement importante que les mesures

se trouvent toutes rassemblées

dans la plage $800-1000^{\circ} \mathrm{C}$. 

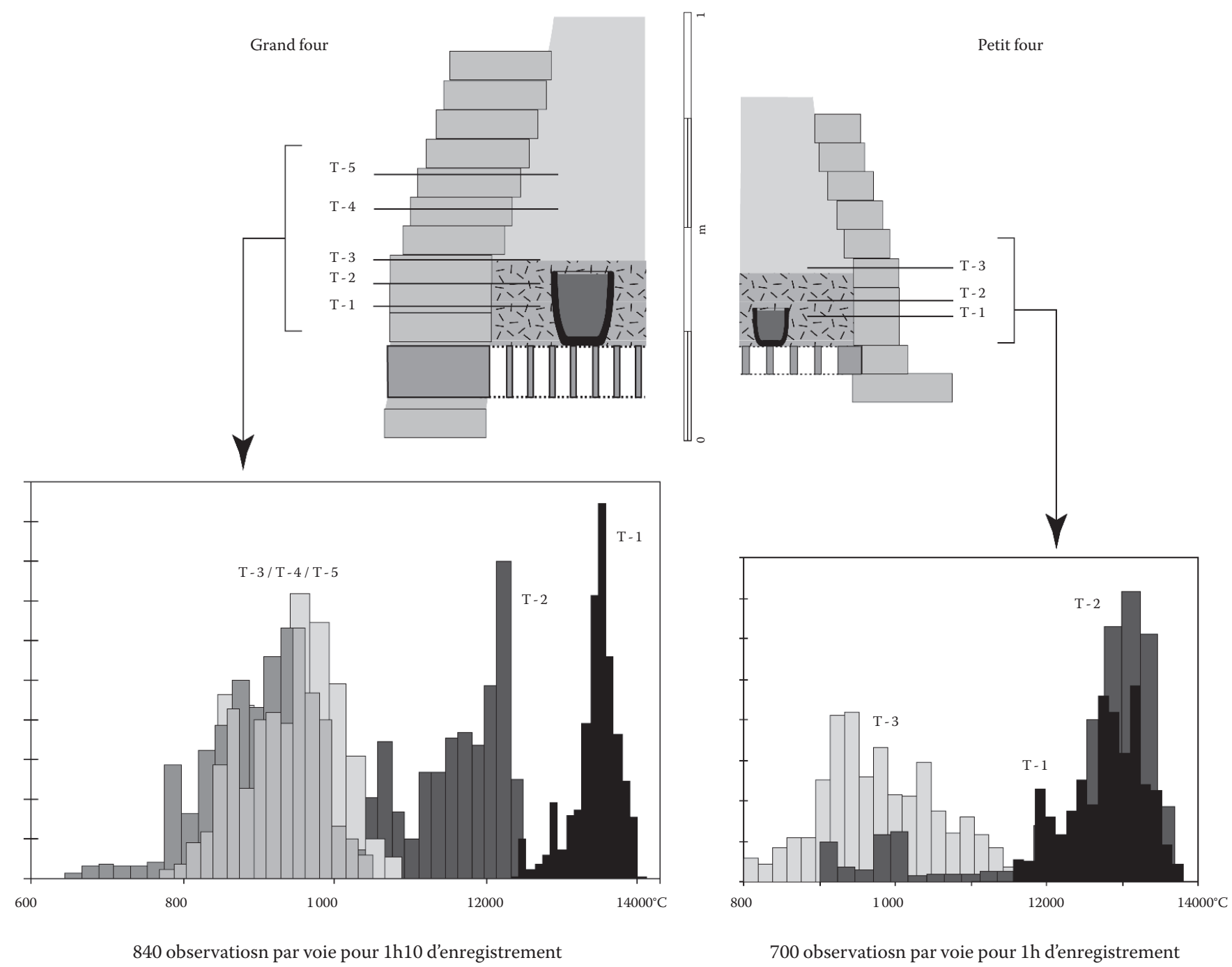


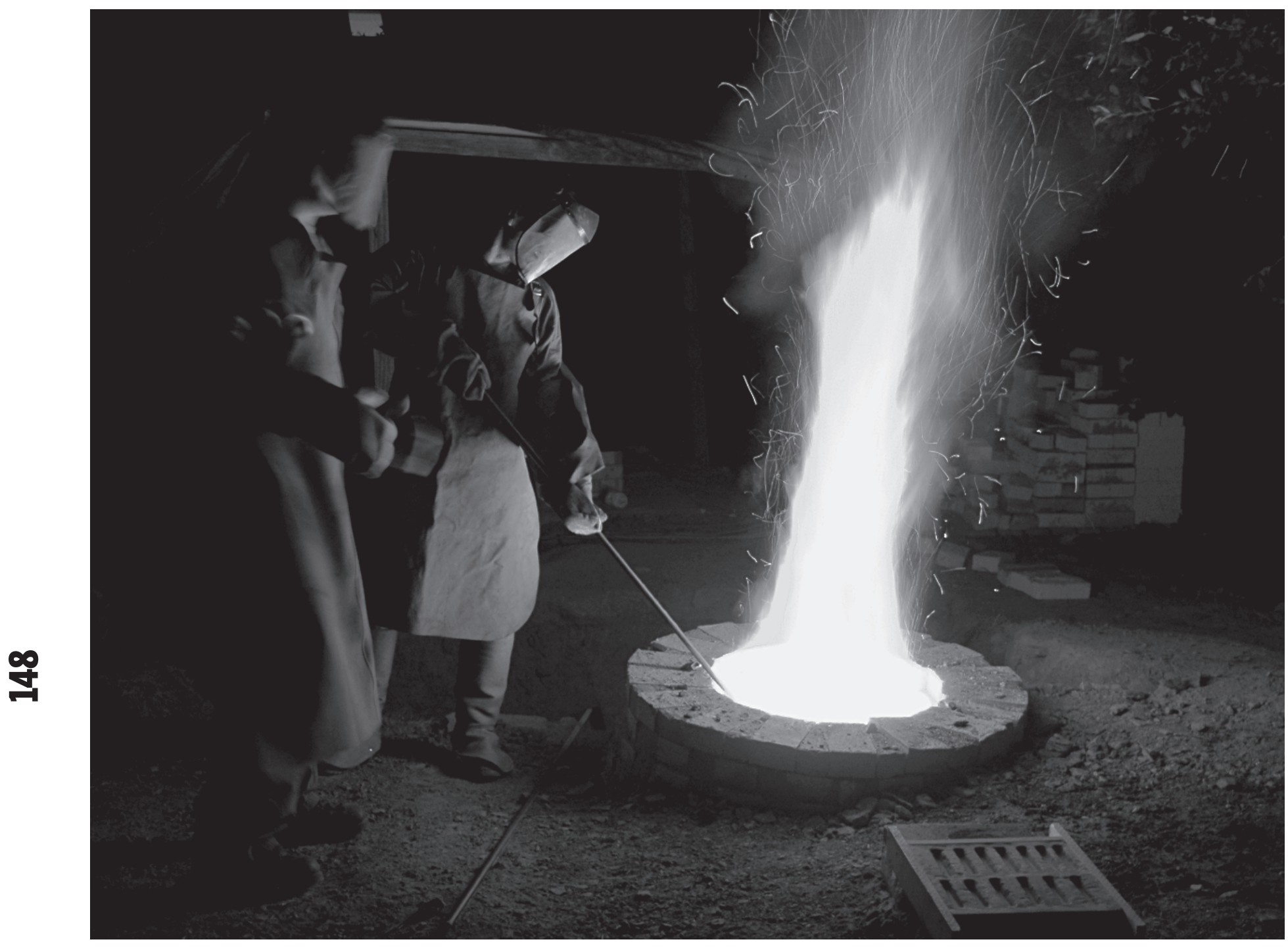

[Fig.5] Photographie du grand four de fusion en fonctionnement. 
de fait accélérée dans un cercle vertueux. Pour atteindre les températures nécessaires à la fusion du cuivre, soit au-dessus de $1100^{\circ} \mathrm{C}$, les paramètres architecturaux de tels fours sont déterminants : diamètre du cylindre, hauteur du four, diamètre et nombre des perforations de la sole, rétrécissement du cylindre [Fig.3]. À ces données, il faut ajouter la dimension des creusets et le calibre du combustible, ces deux dernières variables influant de manière significative sur la bonne marche de ces fours.

L'expérimentation française récente de reconstitution d'un four à partir d'une structure fouillée dans le site parisien a validé ce modèle. Non seulement les températures peuvent être suffisantes, mais les artéfacts produits, comme les déchets de fours, sont très proches des observations archéologiques, ce qui est suffisant pour valider la pertinence du modèle proposé. D'autre part, ces études ont montré comment l'expérimentation pouvait être utile à la fois à l'archéologie, puisqu'elle vise à restituer des élévations disparues, à l'histoire des techniques, en replaçant ces structures dans un système technique plus large, et à l'histoire économique de la production en fournissant des données autrement inaccessibles comme la productivité ou la consommation en combustible. Toutefois, si les fours wallons rentrent incontestablement dans ce modèle, certains posent des questions d'interprétation à cause de leurs dimensions qui s'accroissent, du moins dans certains ateliers : ainsi au XV $\mathrm{XV}^{\mathrm{e}}$ siècle, la fondation des fours est de plus en plus en plus profonde; $\mathrm{au} \mathrm{XIV}^{\mathrm{e}}$ siècle, c'est le diamètre qui augmente. Dans les deux cas, l'archéologie seule ne peut en expliquer les raisons et ne peut rendre surtout compte des implications d'une telle évolution sur les techniques et le rendement.

Du modèle à l'atelier métallurgique. En Wallonie, le programme a conclu un partenariat avec Archéolo-J, une association dont la vocation est la formation et la diffusion de l'archéologie auprès d'un public jeune et étudiant, et un industriel, FIB Belgium, qui apporte un mécénat technologique ${ }^{3}$. Cet ancrage a permis de disposer d'un cadre autorisant la construction de deux fours de fusion de différentes dimensions et leur fonctionnement pendant une semaine à la fois pour réaliser des opérations de fonderie et pour élaborer du laiton. Les paramètres architecturaux ont été définis afin de tester le modèle avec des dimensions se situant aux deux extrêmes du système en fixant le plus de paramètres possibles à l'aide des sources archéologiques ou écrites [Fig.4]. Un grand four, avec un diamètre de $90 \mathrm{~cm}$ et une profondeur de $190 \mathrm{~cm}$ par rapport au niveau du sol, a été reconstruit en se calquant le plus possible sur l'un des fours bouvignois du $\mathrm{Xv}^{\mathrm{e}}$ siècle. Les paramètres inconnus, tels que la hauteur de la chambre de combustion, ont été choisis en fonction des expériences françaises. Le second four, plus petit, a été construit en suivant les indications d'un traité technique du XII ${ }^{\mathrm{e}}$ siècle décrivant le procédé d'élaboration du laiton à la calamine. Les résultats d'une dizaine d'expérimentations avec les deux fours sont nombreux. Globalement, les deux structures ont permis d'atteindre des températures suffisantes, que ce soit pour fondre du cuivre, des alliages ou pour réaliser du laiton. Avec des outils adaptés et des protections en cuir, il n'est pas impossible de travailler à l'intérieur du grand four, malgré l'impressionnante flamme de plusieurs mètres de hauteur qui s'en dégage [Fig.5].

\section{Retour vers les sources archéologiques.} Toutefois, les enseignements les plus significatifs ne sont pas là. Ce modèle de four possède le défaut de présenter un fort gradient thermique vertical dans toute la hauteur de la chambre de combustion où sont déposés les creusets. Pour le petit four, les températures dans la hauteur utile, qui correspond à l'épaisseur du charbon de bois, sont relativement identiques [Fig.4]. On observe toutefois que le plus haut thermocouple a enregistré des températures parfois plus basses, phénomène cyclique dû aux chargements de combustible, alors que les mesures au plus proche de l'arrivée d'air sont beaucoup plus régulières, même si l'on devine plusieurs classes dans la distribution des valeurs. En revanche, dans le grand four, les mesures prises dans l'épaisseur du combustible montrent un gradient très net de $150^{\circ} \mathrm{C}$ sur $5 \mathrm{~cm}$ de hauteur seulement. Or les creusets utilisés sont sous-dimensionnés, avec une vingtaine de centimètres de hauteur, par rapport aux creusets retrouvés à Dinant et à Bouvignes, puisque la plupart ont une hauteur autour de $40 \mathrm{~cm}$. La hauteur du grand four est sans aucun doute trop faible pour obtenir une circulation d'air suffisamment rapide : l'air se trouve freiné en traversant la couche de combustible et n'alimente plus suffisamment la partie supérieure. Avec un four si profondément enfoui, il est possible d'augmenter encore la hauteur de la cheminée sans qu'il soit trop difficile de manipuler les creusets à l'intérieur. C'est ce qui explique en partie que les fours mosans soient très enfouis. Autre raison : en réalisant des opérations de fabrication de laiton dans des creusets au diamètre différent, il apparaît que plus le diamètre du creuset est important, plus le temps de chauffe doit être long pour un enrichissement en zinc comparable. L'inertie thermique des creusets explique pourquoi les hommes du Moyen Âge ont cherché à augmenter la hauteur de leurs récipients sans en modifier le diamètre, afin d'accroître la quantité de métal traité. L'accroissement de la hauteur des creusets a donc entraîné une adaptation des fours. Ces questions en posent d'autres sur la productivité. Pour un gros four, à partir de $70 \mathrm{~cm}$ de diamètre et des creusets d'une vingtaine de centimètres de hauteur, on peut estimer qu'il est possible de fondre plusieurs centaines de kilogrammes en une seule journée (Thomas, 2009, p. 800-804). En augmentant la hauteur des creusets et en adaptant les fours, cette capacité peut largement être décuplée sans pour
Les expérimentatic à une trentaine de kilomètres de Namur. 


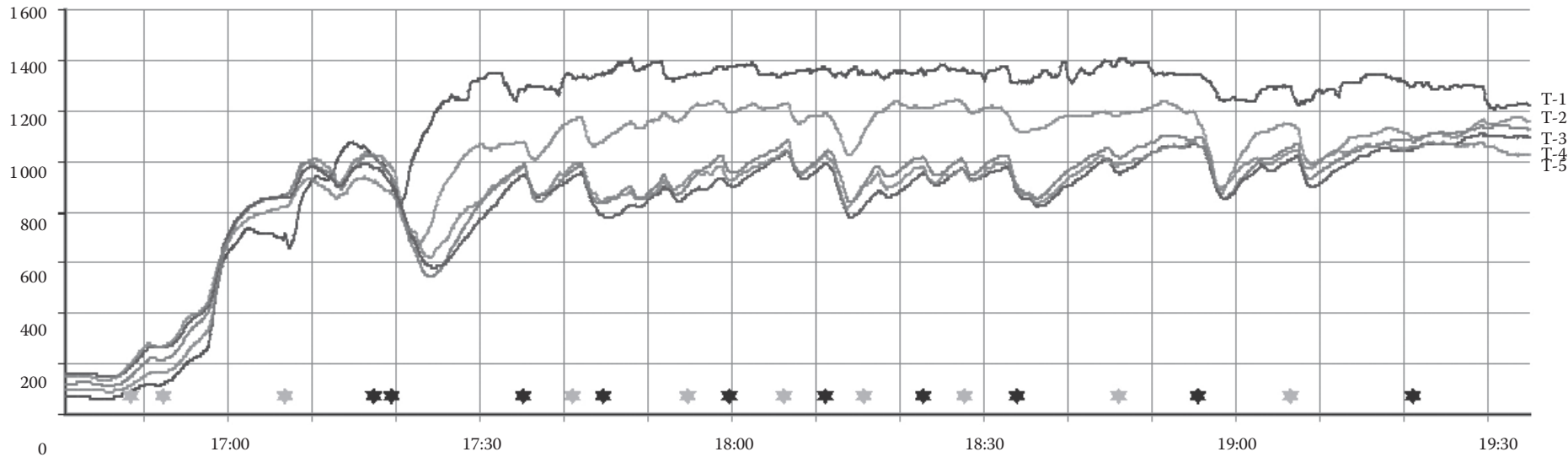

fins de sac de $10 \mathrm{~kg}$ de charbon de bois autres chargements de charbon de bois

[Fig.6] Courbes de températures enregistrées sur le grand four le 16 juillet 2010. Les oscillations affectent quasiment tous les thermocouples lors des chargements en combustible et le gradient thermique vertical. La consommation en combustible est d'environ $50 \mathrm{~kg} / \mathrm{h}$ en moyenne, ce qui correspond à une coulée de plusieurs creusets pour du cuivre non allié et au minimum deux pour des alliages (bronze ou laiton), et ce pour les creusets utilisés. 
autant multiplier la consommation en combustible par le même facteur. En effet, si le petit four brûle quatre fois moins de combustible que le grand, dans les conditions expérimentales testées, et même en multipliant la consommation dans le grand four par deux ou trois après ajustement de sa hauteur, on obtiendrait toujours un rendement nettement meilleur. Ces questions sont au cœur des futurs travaux expérimentaux, car une fois le système technique des ateliers compris et les modèles ajustés, les objectifs sont de préciser les rendements et la productivité des ateliers afin de confronter ces données aux formes du marché.

Un partenariat sur la durée. Le programme de recherche sur la dinanderie mosane s'inscrit dans la durée du fait de la richesse des sites archéologiques et des problématiques posées. Les phases préliminaires d'enregistrement des données ne sont d'ailleurs pas encore achevées, la principale difficulté étant liée à la quantité du mobilier et à son traitement après inventaire. Les études en cours visent à préciser les techniques de fonderie, notamment des chaudrons, et à caractériser les matériaux utilisés comme les terres réfractaires utilisées pour la fabrication des creusets, des moules de fonderie ou encore pour la fabrication des fours. Les analyses de composition d'alliages se poursuivent à un rythme soutenu, tant sur du mobilier belge que français.

Ce projet s'inscrit également dans une collaboration étroite entre l'Inrap et le Service public de Wallonie sur d'autres sujets comme la xylologie $e^{4}$ ou s'insère dans des réflexions stratégiques sur l'archéologie préventive et son évaluation qualitative ${ }^{\mathbf{5}}$. Les proximités géographique et culturelle des deux pays et des enjeux scientifiques souvent communs engagent à poursuivre et à développer des actions de partenariat, sous la forme d'expertises, de collaborations ou d'implication d'agents des deux institutions dans des projets de recherche communs, mais aussi dans le domaine de la formation à l'archéologie préventive.

Bourgarit D., Thomas N., 2009, « Late Medieval copper metallurgy in France: technical insights into the mass production of copper alloy common wares ", in Giumlia-Mair A., Craddock P., Hauptmann A., Bayley J., Cavallini M., Garagnani G., Gilmour B., LA Niece S., Nicodemi W., Rehren T. (ÉD.), Archaeometallurgy in Europe 2007: Selected papers from 2nd International Conference, Aquileia, Italy, 17-21 June 2007, Milano, Associazione Italiana di Metallurgia, CD-ROM.

Collon-Gevaert S., 1951, Histoire des arts du métal en Belgique, Bruxelles, Palais des Académies, Classes des Beaux-Arts, Mémoires, 7, 2 vol., 476 p., 109 pl.

Drescher H., 1982, « Zu den bronzenen Grapen des 12. und 16. Jahrhunderts aus Nordwestdeutschland », in Aus dem Alltag der mittelalterlichen Stadt, Catalogue de l'exposition au Bremer Landesmuseum für Kunst- und Kulturgeschichte (Focke-Museum), 5 déc. 1982 au 24 avril 1983, Bremen, Sturm Druck, p. 157-174

Dungworth D., Nicholas M., 2004, « Caldarium? An antimony bronze used for medieval and post-medieval cast domestic vessels », Historical Metallurgy, 38, 1, p. 24-34.

Pirenne H., 1951a, « Dinant dans la Hanse teutonique », in Pirenne H., Histoire économique de l'Occident médiéval, Bruges, Desclée de Brouwer, p. 501-522.

PirenNe H., 1951b, «Les marchands batteurs de Dinant au XIV ${ }^{\mathrm{e}}$ et au $\mathrm{XV}^{\mathrm{e}}$ siècle : contribution à l'histoire du commerce en gros au Moyen Âge", in Pirenne H., Histoire économique de l'Occident médiéval, Bruges, Desclée de Brouwer, p. 523-531.
Plumier J., Berckmans O., 1996, « Fortifications urbaines à Dinant/ Bouvignes-sur-Meuse », in Plumier J. (DIR.), Cinq années d'archéologie en province de Namur 1990-1995, Namur, ministère de la Région wallonne - Division du Patrimoine, études et documents, série Fouilles, 3, p. 43-49.

Thomas N., 2009, Les Ateliers urbains de travail du cuivre et de ses alliages au bas Moyen Âge : Archéologie et histoire d'un site parisien $d u$ XIV siècle dans la Villeneuve du Temple (1325-1350), Thèse de l'Université Paris 1-Panthéon-Sorbonne, 974 p. et 285 pl.

ToussainT J. (DIR.), 2005, Art du laiton, dinanderie, Namur, Société archéologique de Namur, $392 \mathrm{p}$.

YANTE J.-M., 1988, « La métallurgie urbaine en pays mosan. $\mathrm{XIII}^{\mathrm{e}}$-XVI ${ }^{\mathrm{e}}$ siècle », in BenOIT P., CaIlleaux D., Hommes et travail du métal dans les villes médiévales, Actes de la table ronde, Paris le 23 mars 1984, Paris, AedeH, p. 173-184.
Dietrich sur des cercueils abbatial et paroissial 5 Expertise de Pascal Depaepe, Directeur scientifique et technique de l'Inrap. 